\title{
Carnets
}

Revue électronique d'études françaises de l'APEF

Deuxième série - 13 | 2018

Corps, rythmes et voix : en/jeux littéraires et artistiques

\section{« Ta en tê phonê »}

L'inconnu de la voix dans le langage

Jean-François Savang

\section{(2) OpenEdition}

\section{Journals}

Édition électronique

URL : http://journals.openedition.org/carnets/2631

DOI : $10.4000 /$ carnets.2631

ISSN : 1646-7698

Éditeur

APEF

Référence électronique

Jean-François Savang, " "Ta en tê phonê » », Carnets [En ligne], Deuxième série - 13 | 2018, mis en ligne le 31 mai 2018, consulté le 01 mai 2019. URL : http://journals.openedition.org/carnets/2631 DOI : 10.4000/carnets.2631

Ce document a été généré automatiquement le 1 mai 2019.

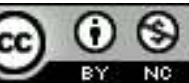

Carnets est mis à disposition selon les termes de la licence Creative Commons - Atribution - Pas d'utilisation commerciale 4.0 International. 


\title{
«Ta en tê phonê »
}

\author{
L'inconnu de la voix dans le langage
}

Jean-François Savang

"je passerai ma vie à ressembler à ma voix ${ }^{1}$ "

1 «Ta en tê phonê » signifie littéralement «ce qui est dans la voix » (16a 3) et se situe au début du Peri hermênias. En nous focalisant sur l'expression Ta en tê phonê, nous voulons mettre en évidence l'enjeu théorique de la traduction dans la conception des rapports entre le langage et la voix - entre le langage et le corps. En effet, si les traductions font passer des voix dans d'autres voix, elles sont aussi « effaçantes ", au sens où, selon Henri Meschonnic « la traduction selon le signe (...) efface le continu (...) et elle efface son propre effacement. (...) ainsi l'Occident se fonde sur l'effacement même de ses origines » (Meschonnic, 2007 : 46). Ce n'est pas un attachement excessif au détail ou à l'insignifiance qui conduit la critique de la traduction par Meschonnic, mais bien la question de l'implication de la traduction dans les « théories du sens».

2 Il ne s'agit pas dans cette réflexion de chercher une origine de la voix, mais plutôt de s'interroger sur le caractère flottant et même historique de la voix dans son rapport au langage. Nous allons le voir, l'indétermination de la voix dans le texte d'Aristote ressortit à des enjeux problématiques plus larges que ceux d'un traduire technique de la langue. C'est toute la question des rapports entre corps et langage qui est posée à partir de l'inconnu dans la voix; en témoigne Porphyre du côté du langage qui rapporte « qu'il y avait un doute dans les opinions des philosophes anciens [à propos] de ce qui est proprement signifié par les mots » (Mora-Márquez, 2011 : 69) et, par extension, sur la place à accorder à la voix dans le rapport entre corps et langage.

\section{Situation}

Le Peri hermênias d'Aristote (384-322 av. J.-C.) est le deuxième livre de l'Organon. Traduit traditionnellement par «De l'interprétation», le Peri hermênias (Hermêneia) a été abondamment commenté pendant l'Antiquité. Cependant, de cette première somme ne nous restent que des témoignages épars ou de seconde main; il se passe près de huit siècles avant les premiers commentaires qui nous sont parvenus. Ammonius fils 
d'Hermias (vers 440-517), puis Boèce (vers 480-524) et plus tard Thomas d'Aquin souligneront l'obscurité du traité d'Aristote, notamment du fait de sa concision; un dicton cité par Cassiodore rapporte que, dans ses Institutions, « Aristote, en écrivant le Peri hermeneias, encrait sa plume dans sa pensée.» (Isaac, 1953: 12) Porphyre également mentionne cette même difficulté de lecture chez des commentateurs antérieurs. Pourtant nombreux ont été les commentateurs, qu'ils fussent Grecs, Syriaques (Probus), Arabes (Al Farabi, Avicenne, Averroès) ou Latin (Victorinus, Boèce). En outre, c'est l'exposé en grec d'Ammonius (fils d'Hermias) au ve siècle qui constituera principalement « la base de tous les travaux grecs ultérieurs » (Isaac, 1953: 13), et cela jusqu'à la traduction en latin que Guillaume de Moerbeke fit pour Thomas d'Aquin au XIII siècle : «Sunt quidem igitur quae in voce, earum quae in anima passionum symbola, et quae scribuntur et eorum quae in voce " (Isaac, $1953: 160)$; en 1268 Moerbeke traduit Ta en tê phonê par « quae in voce ». Si Thomas d'Aquin s'interroge sur le fait qu'Aristote ne donne pas d'indice sur le rapport entre voix et discours, c'est selon lui parce que «la voix est un élément du monde physique (...) [et] c'est pourquoi la voix ne peut être considérée comme le genre du discours " (Verbeke, 1961: XXVII). Les commentateurs [latin] ont eu bien du mal à expliquer pourquoi Aristote avait écrit "ta en tê phonê", considérant qu'il fallait restituer la valeur neutre de l'expression (ce qui est dans la voix).

Dans le dernier chapitre du Peri hermênias (23a 30-31), Aristote utilise la même expression

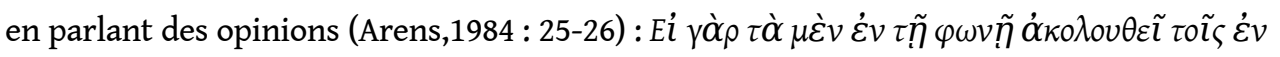

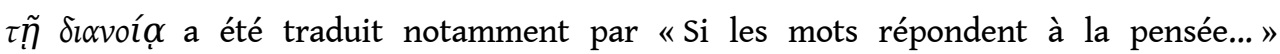
(Barthélémy-Saint-Hilaire, 1844: 199), " Si, en effet, les sons émis par la voix accompagnent ce qui se passe dans l'esprit... » (Tricot, 1997 : 138) « Si l'on admet que ce qui est du domaine du son vocal suit ce qui est dans le mouvement de pensée...» (Dalimier, 2007 : 325). L'expression kiờ $\delta \dot{\varepsilon} \alpha i \dot{\varepsilon} v \tau \tilde{\eta} \varphi \omega v \tilde{\eta}$ (24b 2-4) pour parler des affirmations et des négations est reprise un peu plus loin dans le dernier chapitre : «les affirmations et les négations proférées par la voix sont les symboles de celles [des choses] qui sont dans l'esprit» (Tricot, 1997 : 143). Selon Barthélémy-Saint-Hilaire, Ammonius mettait en doute l'authenticité de ce dernier chapitre ; pourtant, ce n'est peut-être pas un hasard - quelles qu'en soient les raisons - si on retrouve l'expression $\tau \grave{\alpha} \mu \dot{\varepsilon} v \dot{\varepsilon} v \tau \tilde{\eta} \varphi \omega v \tilde{\eta}$ au début et à la fin de l'ouvrage d'Aristote. La voix encadre le rapport au langage comme le corps prend au langage la dynamique tant du rapport aux choses qu'à leur invention. Une certaine symétrie découle de cet inconnu de la voix dans le rapport au langage.

\section{La voix, premier interprétant}

5 La voix serait-elle inséparable de l'organisation du sens? C'est ce que semble suggérer Paul Ricoeur en référence à la conception de l'interprétation dans le traité d'Aristote. La voix serait intimement liée à l'activité discursive et à ce titre on ne pourrait pas séparer radicalement l'activité de la voix de celle du langage. En effet, la voix contribue à l'ordonnancement corporel du discours, à l'image de l'implication mutuelle de la vie et du langage comme des formes-sujets. Elle est engagement dans le sens porté par un sujet :

Est interprétation tout son émis par la voix et doté de signification - toute phồè semantikè, toute vox significativa. En ce sens le nom est déjà par lui-même interprétation et le verbe aussi, puisque nous y énonçons quelque chose; mais renonciation simple ou phasis est prélevée sur le sens total du logos; le sens 
complet de l'Hermêneia n'apparaît donc qu'avec l'énoncé complexe, avec la phrase, qu'Aristote appelle logos. (Ricoeur, $1965: 31$ )

6 Depuis Aristote jusqu'à aujourd'hui la notion d'interprétation apparaît donc directement reliée à la voix. Elle serait l'interprétant entre les mots et les choses dans le langage mais aussi, d'une autre manière, l'articulation des affections de l'âme et de l'activité signifiante de la pensée. Selon Pierre Aubenque, «La voix est ce qui en premier lieu traduit ('interprète') la représentation » (Aubenque, 2009: 40). Autrement dit la voix fait passage des transformations $d u$ sujet en langage; elle implique une vision du monde, une subjectivation.

7 La logique organisatrice du logos passe ainsi par différents niveaux d'interprétation. La notion de voix (phonê/vox) s'inscrit dans une dynamique d'ensemble : à la fois comme indéterminé du sens, à la fois comme émanation physique voire "pathématique » de l'organisation du sens. Autrement dit la voix est liée à l'activité du langage en tant qu'elle symbolise le passage de l'âme au sens, à travers le corps. Il y aurait donc une oralité première du langage qui passe tant par la voix que par la diffusion des affections de l'âme dans l'écrit. Pour Boèce, par exemple, « une interpretatio est un son vocal signifiant, capable de signifier autre chose par lui-même» (Husson, 2009: 25-26); la notion d'interprétation a non seulement un sens beaucoup large qu'aujourd'hui, mais en plus elle mobilise tant la question du corps que la question de l'âme dans le rapport au monde. Subsiste dans cette distribution inédite de l'interprétation un rapport à l'inconnu du sens, au langage comme potentialité du monde et du sujet et non pas simplement une opération technique ou strictement logique au sens où nous l'entendrions aujourd'hui. Le continu du langage évoqué dans le Peri hermênias, sa force de symbolisation, mobilise aussi la transformation du langage intérieur en déclaration du sujet au monde, en inscription signifiante. Il y aurait là l'évocation d'un langage à des états différents de symbolisation allant de l'inconnu des affections de l'âme à l'inscription grammatologique : l'analyse d'une logique indissociable d'une métaphysique. D'où peutêtre un faire interne au traité d'Aristote, montrant le langage, de la situation de l'obscur à son activité d'élucidation. La primauté de la voix par rapport au son et à l'écrit tiendrait au fait que la «La voix est, en un certain sens, vague et indéterminée dans la parole : de son côté, la lettre trouve son origine dans la forme et non l'inverse. L'écriture découle de la voix plutôt que le contraire. (...) Aristote n'argumente pas dans la perspective de l'écriture mais bien plus dans celle de la parole » (Falque, 2016:69). La voix n'est jamais seulement le son, le langage, ou le corps; elle est un rapport d'écoute. Elle ne porte pas seulement dans son grain l'identité intime d'un individu, mais elle « éclabousse » au sens des atomes de Démocrite, elle constitue un périple de l'inter- voire de la transsubjectivité. La voix rend le langage flottant : elle est comme la manifestation de la vie dans le langage, ce qui anime le langage et lui donne force.

\section{La voix pathématique}

Dans son analyse du Peri hermênias, Suzanne Husson précise en note une distinction qui pourrait permettre d'affiner l'analyse de la voix: "comme me le signale J. Barnes, Ammonius, en commentant les premières phrases d'Aristote, distingue en plus du logos parlé et écrit, un logos qui est dans l'âme et qui concerne les simples pensées et le dénommé langage, 'intérieur'2 » (Husson, 2009 : note 3, 25). Autrement dit, si le point de vue premier dans le langage est la voix ou l'oralité, alors peut-être Ammonius se posait-il 
déjà, traduit ici en termes modernes, la question de la pénétration mutuelle du langage et du sujet ; la voix constituerait le caractère anthropologique de toute activité du langage.

La notion de voix est liée à l'activité du langage au moins depuis Ammonius et ensuite depuis la tradition instituée par Boèce en termes de " vox significativa ». Si la voix a une portée naturelle, à la différence du langage comme convention, c'est au sens de la physis grecque qui interprète toute chose de la nature en référence à une métaphysique ou à une cosmogonie. La voix impliquée dans l'interprétation caractérise l'être humain à la différence de l'animal dès lors qu'elle engage l'intention de signifier ; la voix serait ce qui déclenche dans le corps les processus de signifiance, la perspective d'une connaissance ou d'une reconnaissance de soi dans le rapport au monde. La voix serait à ce titre l'avènement d'un continu de l'inconnu dans l'organisation du langage, une dynamique de l'invention du sujet en langage. Le lien entre affect et concept qu'on retrouvera plus tard chez Spinoza, semble déjà faire sens dans les conceptions les plus anciennes du langage et notamment ici, dans le traité d'Aristote, à travers la relation entre pathemata et noemata.

10 En ce sens, la voix qu'on reconnaît comme la prise du corps dans le langage est déjà une activité signifiante qui engage le corps dans les enjeux de symbolisation. Cet inconnu des choses qui sont dans la voix porte l'inconnu d'un quelque chose de la voix qui est plus que du langage, un inconnu du sujet propre à la réalisation de la vie dans le langage. Et l'écriture apparaît prise dans cette oralité décisive qui fait que la parole, la voix signifiante, continue des pathemata aux gramma en passant par les noemata et les pragmata ; et cela du début à la fin du Peri hermênias, la voix traverse l'interprétation voire, se poserait comme premier interprétant de la vie du sujet dans le langage.

\section{La phonê animale}

11 Le recoupement avec le passage II-8 du De anima d'Aristote a souvent été invoqué pour justifier le lien intrinsèque entre la voix et le langage. Aristote fait référence à cet égard à la voix comme "son significatif (sémantikos tis psophos)» et non comme son physiologique. Il apparait cependant que certaines traductions ne prennent pas en compte le De anima d'Aristote pour éclairer la notion de voix dans le Peri hermêneias, dès lors qu'elles considèrent que le terme phonê ne s'applique pas aux animaux. La voix, à cet égard, ne pourrait signifier en dehors d'un rapport déterminé au sens : « $\Phi \omega v \eta े$ signifie exclusivement la voix, commune à l'homme et à certains animaux, et genre de la parole; cette dernière est la voix articulée et elle est propre à l'homme » (Tricot, 1988:119). Le périmètre antique du sens de la phonê était, semble-t-il, plus vague et plus large que ce que la voix nous fait entendre aujourd'hui. Il y a bien la voix d'une certaine manière dans le langage, mais ce n'est pas toute la voix. À cet égard, il paraît un peu étrange de rendre voix par «son vocal» et de réduire la phonê à la sonorité de la parole. Sachant que, comme le rappelle Hans Arens ou Georgio Agamben, si la voix est « interprétation »voire le premier interprétant du langage dans le traité d'Aristote - l'hermeneia à la différence de la phonê, justement, ne s'applique pas à une voix hypothétique des animaux.

Aristote distingue donc manifestement le simple «son", psophos, qui n'est pas reconnu comme une " voix ", phônè, du « son significatif ", psophos sémantikos, qui, lui, est proprement une "voix ", à l'aide d'un double critère : pour qu'un "son " (psophos) soit « doué de signification » (sémantikos), il faut que celui qui l'émette soit un "être animé » (empsuchon) et qu'il le fasse "avec une certaine phantasia». (Labarrière, 2004 : 41) 
12 Autrement dit, la voix n'est pas seulement un phénomène physico-sonore, mais elle est aussi ce qu'elle produit, une extension voire un écho de son articulation dans le monde. Plus encore, sa manière en tant que phantasia suggère l'implication d'une subjectivité, une capacité d'invention propre à l'activité humaine à travers la transformation de la pensée du langage en image, à travers les affections de l'âme. S'appuyant sur l'interprétation d'Aristote par Bodéüs, Labarrière déduit du rapport anthropologique entre langage et voix qu'il pourrait s'agir en fait d'une manière de signifier parmi d'autres au sens où les animaux ont une voix inarticulée apte à signifier aussi, au-delà du phénomène sonore ( psophos) : "la voix (phônè) et le son (psophos) sont deux choses distinctes, et le langage articulé (dialektos) en est une troisième » (Labarrière, 2004 : 41). Ainsi, bien qu'ils n'aient pas le langage (dialektos ou herméneia), les animaux aussi, selon Aristote, ont une phonê, c'est-à-dire une capacité non linguistique à manifester une signifiance interprétable par l'homme.

13 Certes, la voix « contient » mais elle est d'abord processus, rapport au monde. Il ne s'agit pas d'en rester à la conception de la voix comme intentionnalité d'un dire, comme identité sonore d'un sujet au langage, mais bien d'en considérer le caractère spécifique au sens où la voix n'est ni le son, ni le langage en soi. Si Aristote fait de la voix un révélateur de la capacité humaine à produire du sens, il ne la réduit pas, en tant que phonề à un caractère strictement anthropologique. Certes, la voix est associée au langage depuis Aristote; et c'est par similitude que le Stagirite élargit les propriétés de la voix aux « choses sans vie, qui ont une vibration, un chant, un langage. Il semble qu'elles aient une voix, parce que la voix a aussi toutes ces nuances » (Barthélémy Saint-Hilaire, 1846 : 223). Chez Homère, à la différence, le terme phonê ne s'appliquait apparemment qu'à la voix humaine.

\section{Le chant du signe}

L'interprétation médiévale de la théorie de la signification d'Aristote, héritée de Boèce, a principalement perpétué la conception de la voix comme signe des affections de l'âme dans le langage: "Aristote a considéré la voix comme étant d'abord significative des affections de l'âme et deuxièmement des choses extérieures à l'âme ${ }^{3}$ » (Magee, 1989 : 17). Depuis, rapporte Norman Kretzmann, les descendants de la traduction de Boèce se représentent mal Aristote «concernant la voix, les impressions mentales et les choses concrètes $^{4}$ » (Magee, 1989 : 18). La voix est ainsi remplacée par ce dont elle serait le signe, comme si elle était le signe du sens, le signe d'une présence-absence du langage. L'effacement de la voix dans les traductions est d'une certaine manière un effacement du sujet et du corps dans le langage, au profit d'une conception logique du langage. L'absence de distinction entre signe et symbole dans la seule conception du signe aura ainsi des effets dans les manières de traduire comme dans les conceptions du monde :

La plupart des théories sémantiques médiévales prennent leur point de départ avec

la traduction par Boèce (...) du chapitre 1 du De Interpretatione d'Aristote. Nul doute que la perpétuation des erreurs d'interprétation de ce passage pendant et après le Moyen-âge ne soit largement due à une fidélité excessive au texte même de Boèce, ce dernier ayant escamoté la distinction aristotélicienne entre symboles et signes en traduisant à la fois symbola et semeia par notae ${ }^{5}$. (Magee, $1989: 48$ )

Dans de nombreuses traductions les deux notions semeia et symbola apparaissent de manière interchangeable. Si dans une perspective sémiotique l'assimilation du signe et du 
symbole ne pose pas de problème, il en va autrement du point de vue de Kretzmann: «Est-ce que les mots signes (semeia) et symboles (symbola) sont synonymes ici? (...) De nombreux traducteurs en sont si évidemment sûrs qu'ils ne donnent même pas au lecteur la possibilité de se poser la question ${ }^{6} »($ Kretzmann, 1974:7). En effet, dans le contexte d'une théorie du langage basée sur la relation de signe, le son et le sens sont presque venus naturellement habiter la voix. L'institutionnalisation du signe et d'une lecture sémiotique du monde seraient déjà formulées chez Aristote avant Augustin : « le langage est signe depuis Aristote : ta en te phone, les " voix » (voces), sont semeia ou symbola depuis De interpretatione. (...) quand, en Grèce, le langage devient semeion. Saint Augustin subsume le verbe définitivement sous le signe » (Trabant, $2002: 270$ ). Il ne s'agit pas d'en conclure à un tournant aristotélicien du signe mais, plutôt, à une lecture historique de l'opposition du corps et du langage ; à l'institutionnalisation philosophique de la voix comme signe sonore d'une ontologie du sujet. C'est sans doute cette détermination de tout le langage dans ce premier universalisme du signe depuis Aristote qui mobilise la critique d'Henri Meschonnic après Humboldt :

Humboldt s'oppose directement à l'interprétation aristotélicienne du mot comme arbitraire du signe. Humboldt dénonce cette conception qui s'est institutionnalisée à travers les siècles: d'abord que la pensée serait universellement la même et qu'elle se réaliserait en dehors du langage et ensuite que les mots sont des instruments de communication de ces pensées, des "formes sonores " (ta en tê phonê) qui sont des signes (semeia) ${ }^{7}$. (Trabant, $2017: 2-3$ )

Nous constatons, à travers les enjeux du signe et du symbole, que la voix, en tant que rapport ou articulation, constitue une question qui déborde sa propre définition: s'interroger sur la voix, c'est alors, comme le fait remarquer Meschonnic, s'interroger sur l'ensemble de la théorie du langage. Comme l'indique par ailleurs Kretzmann, «le langage n'est pas un système de signes sui generis »; c'est-à-dire que la sémiotique n'est pas une nature du langage, même si bon nombre de traductions ont fonctionné sur ce principe d'Augustin à C.S. Peirce - que tout pouvait devenir le signe de tout et, notamment que la voix pouvait devenir tantôt le signe de la pensée dans le langage, tantôt sa manifestation sonore, tantôt l'articulation des deux. Mais peut-être la théorie du langage d'Aristote estelle plus ouverte, paradoxalement, qu'une sémiotique de la lettre appliquée à la voix. En effet, ajoute Kretzmann, la langue « est juste le plus complexe, le plus flexible, le plus riche des modes de signification; cependant plus les modes de signification sont artificiels, nous rappelle Aristote, moins ils sont construits sur des bases artificielles ${ }^{8}$ " (Kretzmann, 1974 : 18). S'appuyant sur Aristote, Kretzmann - à l'instar de Humboldt met en évidence le lien indissociable entre les enjeux techniques du langage et le caractère anthropologique de toute signification.

\section{Critique du gramma}

17 La surdétermination du gramma par rapport au phonê signifie que «la réflexion occidentale sur le langage situe, depuis le début, le gramma et non la voix dans le lieu originel » (Agamben, 1991 : 80-81). En effet, ce n'est pas avec les choses (pragmata) ou avec les concepts (noemata) ou même avec les lettres (gramma) mais avec l'inconnu du langage dans la voix que commence le traité d'Aristote.

La voix incorporerait alors le symbole d'une présence-absence du corps dans le langage, une transparence du sujet dans le langage : elle devient alors emblème d'une institution 
du signe comme le développera Jacques Derrida dans La voix et le phénomène (1967a) ou dans De la grammatologie (1967b). La notion de voix y sert la démonstration du signe. Comme le signale Derrida « le langage est bien le médium de ce jeu de la présence et de absence » (Derrida, 1967a: 9). Pour Derrida, la voix est déjà le signe ontologique d'un idéal du sujet, la manifestation phénoménologique d'une présence. La voix est alors décrite comme un médium de l'être de l'objet, comme signe phonique du monde dans le langage : «la voix est le nom de cet élément. La voix s'entend» (Derrida, 1967a: 85). Derrida ajoute plus loin : « la voix est la conscience» (Derrida, 1967a: 89) et insiste sur l'être du sujet que la voix porterait en se faisant entendre, intérioriser et répéter comme passage d'une " auto-affection pure » (Derrida, 1967a: 89) d'une subjectivité à une autre. À la fin de La Voix et le phénomène Derrida va préciser l'incidence nécessaire à la réalisation de la voix dans le rapport à l'écriture : « Une voix sans différance, une voix sans écriture est à la fois absolument vive et absolument morte » (Derrida, 1967a : 115).

L'écho entre La Voix et le phénomène et De la Grammatologie n'est pas seulement synchronique. Dans De la Grammatologie Derrida fait directement référence au «ta en tê phonê » d'Aristote : il considère l'implication du logos dans la voix comme une seconde nature, comme la condition interne de la vocation signifiante de la voix :

dans ce logos, le lien originaire et essentiel à la phonè n'a jamais été rompu. (...)

l'essence de la phonè serait immédiatement proche de ce qui dans la «pensée » comme logos a rapport au "sens », le produit, le reçoit, le dit, le " rassemble ». Si, pour Aristote, par exemple, « les sons émis par la voix (...) sont les symboles des états de l'âme (...) et les mots écrits les symboles des mots émis par la voix " (De l'interprétation 1, 16a 3), c'est que la voix, productrice des premiers symboles, a un rapport de proximité essentielle et immédiate avec l'âme. Productrice du premier signifiant, elle n'est pas un simple signifiant parmi d'autres. Elle signifie l'« état d'âme ». (Derrida, 1967b : 21-22)

C'est par « ressemblance naturelle», par réduction de la voix au signe ( = ressemblance/ likeness pour C.S. Peirce) que Derrida détermine un lien implicite entre logos et phonê ; il situe ce lien dans un autre rapport - de traduction naturelle - plus fondamental selon lui entre l'âme/l'être et le monde. La théorie du langage que nous livre ici Derrida est celle d'une tradition de la conscience du monde dans son inscription, considérant que les mots sont a priori dans la voix. En effet, selon le philosophe, « les affections de l'âme exprimant naturellement les choses, elles constituent une sorte de langage universel qui dès lors peut s'effacer de lui-même » (Derrida, 1967b : 22). Puisqu'elle est le signe/expression de l'âme, la voix serait non seulement un négatif du corps mais aussi un négatif et une exclusion des règles de l'écrit, instituant le monde dans une sorte de sémiotique spontanée. Derrida l'appelle «l'étape de la transparence ». Certes Derrida, critique le privilège du sonore dans la voix en faisant de la voix un signe de l'être. Cependant, faisant de la voix l'enjeu d'une présence-absence de l'âme et de l'être dans le langage, il fait de ta en tê phonê une question essentiellement métaphysique.

21 Or il apparaît que ce qui fait empreinte, marquage, c'est bien l'inscription de la voix du sujet dans l'écrit. Prend tout son sens, ici, l'enjeu d'un continu de la voix, tant à l'oral qu'à l'écrit. S'il devait y avoir une matière du sens voire une physique du langage, ce serait la voix qui lui donne son empiricité première et non l'écriture.

Les formes écrites seraient-elles les symboles de ce qui est dans la voix ? Ce serait réduire la voix à la signifiance de la langue là où elle excède nécessairement les mots comme agencement de l'esprit dans le corps. "Ce qui est dans la voix» est une puissance de signifiance indéterminée en tant que telle, un infini de la capacité à produire du sens et 
de l'inconnu dans le langage. La preuve en est sans doute le fait qu'Aristote octroie une voix aux animaux. "Ce qui est dans la voix » serait déjà, en quelque sorte, d'un point de vue rhématique, la capacité générique du sujet à s'inventer dans le verbe, mais aussi la base d'une inter- et d'une transsubjectivité, d'un fonctionnement de l'interprétation, c'est-à-dire d'une interprétance. La voix en ce sens est action d'un corps-langage de la pensée. Ce qui est dans la voix ne serait-ce pas alors la pensée au sens d'un continu du sujet, l'articulation d'une plasticité propre à l'articulation de la pensée dans le corps et au corps dans la pensée? Une energeia de rapports transitoires constituant justement le langage dans la voix comme une forme-de-vie?

\section{Qu'est-ce qu'il y a dans la voix?}

En fait, en tant que signe, la lettre (gramma) présuppose à la fois la voix et son effacement - et en particulier, l'effacement du corps dans le langage. Au lieu que l'écrit constitue la trace ou l'empreinte du sujet dans le langage, il semblerait que ce soit l'inverse qui s'est généralisé : la matérialité écrite du langage fait signe pour le corps absent et c'est la voix qui apparaît alors comme trace sonore, voire comme transparence du corps dans le langage. D'où le retour à la question et le retournement de la question de l'écrit, du point de vue de la voix : «Qu'est-ce qui est dans la voix » s'interroge Georgio Agamben à partir d'Aristote?

Que sont ta en tê phonê ? Aristote, en effet, énonce en ces termes le processus de signification du discours humain : "Ce qui est dans la voix constitue le symbole des pathèmes de l'âme, et ce qui est écrit, le symbole de ce qui est dans la voix. Et de même que les lettres ne sont pas les mêmes chez tous les hommes, les voix ne sont pas non plus les mêmes, bien que les pathèmes dans l'âme, dont elles sont les signes immédiats, soient identiques chez tous, comme sont identiques aussi les choses dont ces pathèmes sont les images". (De int. 16A, 3-7.) (Agamben, 1991 : 79)

La question se pose également, suite à la remarque d'Henri Meschonnic concernant la même phrase d'Aristote et sa traduction notamment par Victor Cousin comme «les sons qui sont dans la voix ». Or la traduction littérale est beaucoup plus vague et ménage quelque chose de plus énigmatique que du sonore dans la voix : ce serait tout simplement "ce qui est dans la voix». Bien sûr, la question d'une traduction trop littérale peut paraître problématique, dans la mesure où chaque terme d'un texte renvoie au système du texte dans son ensemble. Comme le signale Suzanne Husson: «Dans une traduction très (trop?) proche du grec nous aurions : "les items qui sont dans la voix sont symboles des affections dans l'âme et les items écrits de ceux qui sont dans la voix" " (Husson, 2009: 28 , note 3). Cette dernière réflexion permet de mettre en évidence qu'il ne s'agit pas ici d'un impensé de la traduction; ce sont des choix culturels et philologiques qui ont présidé à l'historicité de la traduction de ta en tê phonê.

Emmanuel Falque défend également une traduction assez littérale de ce passage d'Aristote :

L'expression grecque d'« état de l'âme dans la voix " (pathemata en tê phone) dans le De interpretation d'Aristote, met moins l'accent sur le son émis par la voix - comme si la voix, en soi, était un simple instrument servant à communiquer avec l'âme que sur la "place" ou ce "quelque chose " d'où ces affects se manifestent et s'exprimeraient eux-mêmes plus profondément. (Falque, 2016:67) la voix pour Aristote contiendrait l'âme et c'est seulement après éventuellement qu'elle 
prendrait forme dans un second temps dans l'écrit. À l'opposé de Derrida, pour lequel l'écriture de la voix ou le gramma est central, il serait préférable de mettre en évidence le premier terme : à savoir le pathemata "dans la voix" comme symbole de l'état de l'âme, faisant du sujet un enjeu non linguistique de la réalisation du langage.

First of all, the voice contains thus the pathos, only to afterward and eventually affix it in a written text as its possible, but only secondary, target. In opposition to Derrida, for whom the second term of the opposition - the writing of the voice or the gramma - is central, today it is appropriate to point out the primacy of the first: the pathemata 'in the voice' as symbol of the states of the soul (Falque, 2016: 68).

Parler de "son", de "chose» ou d'un inconnu du langage dans la voix n'est pas équivalent ; la différence première, c'est peut-être qu'il n'y a pas forcément que du sonore dans la voix. C'est un point de vue qu'Agamben partage avec Meschonnic : «La voix (...) n'est pas simplement la phonê, le simple flux sonore émis par l'apparat phonatoire, de même que le $J e$, le locuteur, n'est pas simplement l'individu psychosomatique d'où provient le son » (Agamben, 1991 : 74). Et de même nous pouvons sans doute ici attribuer à la voix ce que Meschonnic dit du rythme, à savoir que «ce n'est pas du son mais du sujet. »

\section{La voix, c'est du sujet}

Il est particulièrement éclairant de la part de Georgio Agamben d'insister sur le caractère à la fois déictique et énonciatif de la voix. Il rappelle, en référence à Émile Benveniste, que les verbes (rhema) dont Aristote fait l'analyse dans le Peri hermênias font partie du système énonciatif et, à ce titre, qu'ils participent de l'implication du sujet dans le discours : "L'énonciation et l'instance de discours ne peuvent être identifiées comme telles qu'à travers la voix qui les profère» (Agamben, 1991: 68) et il souligne à cet égard la clairvoyance du poète Paul Valéry : "Je ou moi est un mot associé à la voix » (ibid.). La voix telle que la décrit Agamben est ici très proche d'un sujet de l'énonciation voire de la subjectivation particulière $d u$ « sujet du poème » de Meschonnic; cependant avec la voix nous sommes au-delà de l'énonciation strictement linguistique, au-delà de la question technique d'un locuteur.

Jean-Louis Labarrière (2004 : 41-42) met en évidence chez Aristote la distinction entre la " voix » phonê et le « son/bruit » psophos en rappelant l'enjeu du rapport dynamique entre sujet et langage, c'est-à-dire l'enjeu de l'«articulation » ou du "langage articulé » dialektos. L'idée est assez proche de celle d'Agamben, lequel, en référence à Aristote, pose la question d'une " pure voix, non pas, toutefois, simplement comme pur son ni comme sphère de signification déterminée, mais comme porteuse d'une signification inconnue » (Agamben, 1991: 70). La voix est alors surgissement du corps dans le langage, surgissement d'un inconnu du sens dans le corps. Elle est peut-être plus la manifestation du sujet à signifier, un désir de langage, qu'une forme du langage en soi. Et sur le plan de cet inconnu du langage dans sa formation, la traduction de phonê en "parole » est ici problématique par rapport à la notion d'epos. La "pure voix» ne relèverait pas à cet égard du sonore mais de l'individualité particulière qui se manifeste dans le sens; autrement dit, pour reprendre le terme d'Henri Meschonnic il s'agirait d'une «formesujet», de «la transformation mutuelle d'une forme-vie en forme-langage. Dans cette perspective, nous comprenons mieux la distinction que Meschonnic fait entre le sujet et le sonore, du point de vue du rythme et nous pourrions l'appliquer à la voix. Et si « ce qui 
est dans la voix » ressortit à l'indétermination du langage du point de vue du sujet, le démonstratif $c e$ renvoie aussi à une détermination infinie du sujet dans le langage, ni son ni parole encore mais déjà signifiance et corporalisation du discours. La voix est sur un plan déictique «l'indication que le langage a lieu » pour un sujet indéfini : c'est montrer le sujet dans son fonctionnement et non dans ce qu'il est.

S'appuyant sur l'expérience de saint Augustin, Agamben identifie alors une "pure intention de signifier » en précisant que «cette expérience d'un verbe inconnu (verbum incognitum) ancré nulle part, entre son et signification, est, selon Augustin, l'expérience amoureuse comme volonté de savoir » (Agamben, 1991 : 71-72). Si la voix est caractérisée par ce qu'on met dedans - du corps, du sujet, du langage, de l'intention, de l'inconnu ? on ne saurait cependant l'aborder selon une stricte réduction ontologique sans envisager la force d'articulation qui fait de son activité un lieu de passage qui n'est ni corps ni langage en soi. D'où la nécessité de lire la question du ta en tê phonê en référence également au Traité de l'âme d'Aristote et en tenant compte d'un caractère de la phonê distinct du psophos et du dialektos. La voix en ce sens correspondrait à la signifiance d'un « non encore signifié »; elle n'est pas « rien » de constituer le transfert du sujet en corpslangage, c'est-à-dire de constituer la part extra-linguistique nécessaire à toute réalisation de la parole en langage, la part non linguistique au sens où « ce qui articule la voix humaine en langage est une pure négativité » (Agamben, 1991 : 75) ou du moins la place qu'il faut faire en creux à la pensée pour qu'elle devienne une forme-de-langage, un négatif ou un extrait du corps dans la voix. D'une certaine manière, si comme l'interrogeait Michel Foucault, le corps est utopique, il y a une dimension de ce corps à travers la voix qui fait à la fois sa réalisation et son non-lieu dans le langage. Sur ce modèle, la voix constituerait l' energeia d'un corps-langage du sujet; le sujet comme «lieu non-lieu» d'une corporalisation dans le langage, pour reprendre l'expression qui définit l'utopie pour Meschonnic.

\section{Conclusion}

31 Selon Agamben, «À la question : "qu'est-ce qui est dans la voix ?", la philosophie répond : rien n'est dans la voix, la voix est le lieu du négatif, elle est Voix» (Agamben, 1991: 81). Selon nous, cependant, la voix ne détermine pas seulement l'expérience du négatif au sens ontologique. Car au « rien » nous associons plutôt la voix à l'inconnu qu'au négatif, c'est-à-dire à l'energeia de l'expérience concrète d'un continu du monde dans le langage par un sujet. « $\mathrm{Ce}$ » ou « rien » dans la voix sont des puissances du langage dans le vide, des dynamiques de la réalisation du monde dans l'inconscient du langage. Ce sont déjà des formes-de-vie, impliquant un infini du monde dans l'inconnu du langage. Les « échanges corporels-langagiers " (Meschonnic, 1982: 654) portés par la voix constituent ainsi ce point de vue si particulier de «ce» qu'on appelle «sujet» comme point de convergence de l'expérience du monde et du langage dans son entier. 


\section{BIBLIOGRAPHIE}

AGAMBEN, Georgio (1991). Le Langage et la mort - un séminaire sur le lieu de la négativité. Paris :

Christian Bourgois Éditeur.

ARENS, Hans (1984). Aristotle's Theory of Language and its Tradition. Texts from 500 to 1750.

Amsterdam/Philadelphia: John Benjamins Publishing.

ARISTOTE (1844). « Hermeneia », Logique d'Aristote, traduit par J. Barthélémy Saint-Hilaire. Paris :

Librairie de Ladrange.

ARISTOTE (1846). Traité de l'âme, livre 2, chapitre VIII, traduit par J. Barthélémy Saint-Hilaire.

Paris : Librairie de Ladrange.

ARISTOTE (1988). De anima [1934], traduit par Jules Tricot. Paris : Vrin.

ARISTOTE (1994). « De l'Interprétation », Organon, traduction et notes par Jules Tricot. Paris : Vrin.

ARISTOTE (2007). Sur l'interprétation. Traduction, présentation et notes par Catherine Dalimier.

Paris : Garnier Flammarion.

AUBENQUE, Pierre (2009). «Sens et unité du traité aristotélicien De l'interprétation », Interpréter le De interpretatione (dir. Suzanne Husson). Paris : Vrin.

DERRIDA, Jacques (1967). De la grammatologie. Paris : Minuit.

DERRIDA, Jacques (1967). La Voix et le phénomène. Paris : PUF, Quadrige.

FALQUE, Emmanuel (2016). «For a Hermeneutic of the Body and the voice », Crossing the Rubicon: the Bordelands of philosophy and theology. New-York : Fordham University Press.

HUSSON, Suzanne (2009). «Introduction », Interpréter le De interpretatione. Paris : Vrin.

ISAAC, Jean (1953). Le Peri hermeneias en Occident de Boèce à Saint Thomas - Historie littéraire d'un traité d'Aristote. Paris: Vrin.

KRETZMANN, Norman (1974). « Aristotle on spoken sound significant by convention », Ancient Logic and Its Modern Interpretations: Proceedings of the Buffalo symposium. 21-22 avril 1972. Boston USA: Edited by John Corcoran, Reidel publishing compagny.

LABARRIÈRE, Jean-Louis (2004). Langage, vie politique et mouvement des animaux - études aristotélicienne Paris: Vrin.

MAGEE, John (1989). Boethius on Signification and Mind. Leiden (Netherlands): E. J. Brill.

MESCHONNIC, Henri (1972). Dédicaces proverbes. Paris : Gallimard.

MESCHONNIC, Henri (1982). Critique du rythme - anthropologie historique du langage. Lagrasse :

Verdier.

MESCHONNIC, Henri (2007). Éthique et politique du traduire. Lagrasse : Verdier.

MORA-MÁRQUEZ, Ana María (2011). «Peri hermeneias 16a3-8. Histoire d'une rupture de la tradition interprétative dans le bas Moyen Âge ", Revue philosophique de la France et de l'étranger, tome 136, n $\circ 1$. 
RICOEUR, Paul (1965). De l'interprétation - essai sur Freud. Paris : Seuil.

TRABANT, Jürgen (2002). « Signe et articulation », Cahiers Ferdinand de Saussure, 54/2001. Genève:

Droz.

TRABANT, Jürgen (2017). « Vanishing worldviews », Forum for Modern Language Studies, vol. 53, Issue 1, jan.2017, pp. 21-34. <URL :https://doi.org/10.1093/fmls/cqw081>

VERBEKE, Gérard (1961). Ammonius - commentaire sur le Peri hermeneias d'Aristote. Louvain :

Publications universitaires de Louvain.

\section{NOTES}

1. Henri Meschonnic, Dédicaces proverbes, Paris, Gallimard, 1972, p. 15.

2. Suzanne Husson précise dans la même note que « Boèce adoptera également cette conception lorsqu'il distinguera trois types d'orationes : écrite, parlée et pensée ».

3. Ma traduction.

4. Ma traduction.

5. Ma traduction. Magee fait référence à Norman Kretzmann (1974:5).

6. Ma traduction.

7. Ma traduction.

8. Ma traduction.

\section{RÉSUMÉS}

Dans cet article nous allons nous interroger sur la valeur de la notion de voix telle qu'elle a été problématisée depuis le Peri herménêias d'Aristote. Plus précisément, nous allons nous focaliser sur l'expression « Ta en tê phonê » qui signifie littéralement «ce qui est dans la voix ». En effet nombre de traductions déterminent précisément « ce qui est dans la voix » par les « mots », les « paroles", les « sons vocaux », ou les « sons ». Or l'expression de la voix dans le texte d'Aristote fait aussi référence à l'indéfini «ce ", à l'enjeu d'une indétermination du langage dans la voix, comme en témoigne chacun à leur façon Henri Meschonnic et Georgio Agamben. Meschonnic déduit notamment de la critique des traductions de la voix chez Aristote, l'héritage d'une institution du signe qui s'est progressivement étendue à l'ensemble de la théorie du langage.

In this paper, we are going to question the voice as it's represented in Aristotle's Peri hermeneias. To be more exact, we focus on the expression «ta en tê phonê » which means "what is in the voice ». In fact, many translations give « what is in the voice " a determined sense as " words", " spoken sounds ", « sound shapes » or merely " sound ». Nevertheless, voice's expression in the Aristotle's text refers to the undefinite "what ", and therefore raises concerns about language indetermination in the voice, as both Henri Meschonnic and Georgio Agamben said. Meschonnic in particular, deductes from the critical Aristotle voice translation, the legacy of sign institution extended to the whole language. 
INDEX

Keywords : Phonê, sign, translation, interpretation, language

Mots-clés : phonê, signe, traduction, interprétation, langage

\section{AUTEUR}

JEAN-FRANÇOIS SAVANG

(Un. Paris 8)

Jean-Francois.Savang[at]univ-nantes.fr 